Doi: 10.22478/ufpb.1983-1579.2020v13n1.51539 http://periodicos.ufpb.br/ojs2/index.php

\title{
FINALIDADES Y METODOLOGÍAS DE ENSEÑANZA PARA LA FORMACIÓN CIU- DADANA EN CHILE
}

\author{
PURPOSES AND TEACHING METHODOLOGIES FOR CITIZEN EDUCATION IN CHILE \\ Sebastián Quintana-Susarte ${ }^{1}$ \\ Alejandro Rabuco Hidalgo ${ }^{2}$
}

\begin{abstract}
Resumen: Este artículo exhibe los resultados de un estudio destinado a analizar las finalidades y metodologías de enseñanza propuestas para la Formación Ciudadana en el sistema educativo chileno, a partir de la revisión documental de las propuestas oficiales del Ministerio de Educación (Mineduc). Los principales resultados dan cuenta de finalidades que vinculan la Formación Ciudadana con la democracia y la participación, aunque sin referir a propósitos de carácter político. En relación a las estrategias de enseñanza, se constata un enfoque centrado en el estudio de problemáticas de interés público. El análisis realizado permite impulsar el concepto "ciudadanía de puertas adentro", que se vincula a la idea de una educación ciudadana dirigida, en mayor medida, al fomento de la discusión y el debate, en desmedro de una formación destinada a promover la participación y la intervención social, elementos considerados imprescindibles en una educación para la ciudadanía crítica y democrática.
\end{abstract}

Palabras claves: Enseñanza de las Ciencias Sociales, Formación Ciudadana, Profesorado, Ciudadanía crítica, Ciudadanía puertas adentro.

Abstract: This article displays the results of a study aimed at analyzing the aims and teaching methodologies proposed for Citizen Training in the Chilean educational system, based on the documentary review of the official proposals of the Ministry of Education (Mineduc). The main results show the aims that link Citizen Training with democracy and participation, although without referring to political purposes. In relation to teaching strategies, there is an approach focused on the study of problems of public interest. The analysis carried out makes it possible to promote the concept of "citizenship behind closed doors", which is linked to the idea of citizen education aimed, to a greater extent, at promoting discussion and debate, to the detriment of training aimed at promoting participation and social intervention, elements considered essential in an education for critical and democratic citizenship.

Key words: Teaching Social Sciences, Citizen Training, Teachers, Critical Citizenship, Indoors Citizenship.

Resumo: Este artigo mostra os resultados de um estudo destinado a analisar os propósitos e metodologias de ensino propostas para a Formação Cidadã no sistema educativo chileno, com base na revisão documental das propostas oficiais do Ministério da Educação (MINEDUC). Os principais resultados dão conta de propósitos que ligam a Formação cidadã à democracia e à participação, embora sem se referir a propósitos políticos. Em relação às estratégias de ensino, há um foco no estudo de questões de interesse público. A análise possibilitou a promoção do conceito de "cidadania de portas fechadas", que está ligada à ideia de uma educação cidadã destinada, em maior medida, à promoção do discussão e debate, no interesse da formação destinada a promover a participação e intervenção social, elementos considerados essenciais numa educação para a cidadania crítica e democrática

Palavras-chave: Ensino de Ciências Sociais, Formação cidadã, Professores, Cidadania Crítica, Cidadania de portas fechadas.

\section{INTRODUCCIÓN}

1 Profesor de Historia, Universidad Católica de Chile. Máster en Didáctica de las Ciencias Sociales y Doctorando en Educación, Universidad Autónoma de Barcelona. Miembro de Red Chilena de Investigación y Enseñanza de las Ciencias Sociales (REDIECS). E-mail: squintanas@gmail.com. Orcid: https://orcid.org/0000-0002-2346-1657.

2 Profesor de Historia y Ciencias Sociales. Máster en Educación, Pontificia Universidad Católica de Valparaíso. Doctorando en Educación Universidad Autónoma de Barcelona. Miembro de Red Chilena de Investigación y Enseñanza de las Ciencias Sociales (REDIECS). E-mail: arturo.rabuco@gmail.com. Orcid: https://orcid.org/0000-0001-6678-9778. 
La formación ciudadana y la educación escolar forman parte de un binomio inseparable. Sus raíces surgen en un devenir histórico donde conviven con conceptos tan complejos como polisémicos, los cuales son trasladados a la escuela para la construcción de una ciudadanía democrática. En lo concreto la ciudadanía, sin importar sus diversas definiciones, se aprende. Esto permite hablar de una configuración de procesos de aprendizaje ciudadano y enseñanza de lo político (MUÑOZ; GAMBOA; URBINA, 2013, p.128). Gómez (2005) define esto como un proceso de endoculturación política, que sitúa a la escuela como un espacio generador de significados construido desde las diversas interacciones sociales de los sujetos. A partir de estas prácticas se producen y reproducen discursos que dan vida a la forma en que se concibe la ciudadanía y lo político en contextos particulares.

La conceptualización antes referida pone de relieve dos ámbitos inseparables de la formación ciudadana: lo político y la educación. Se trata de comprender que la educación para la ciudadanía no puede ser un fenómeno situado desde lo abstracto, sino que su legitimación proviene desde la comunidad en donde se ejerce. Por ello, la formación ciudadana al ser un aprendizaje situado tiene directa relación con las características culturales y sociales de los contextos educativos. Vale decir que, la subjetividad de profesores y estudiantes, no puede ser reducida a la imagen del títere social que entiende la participación política en correlación exclusiva del sufragio electoral. Es por eso que la educación para la ciudadanía es comprendida, desde el pensamiento de FREIRE (2006), como una forma de intervención en el mundo que posibilita a los sujetos y a la escuela a asumir un rol histórico.

Por tanto, la forma en que se enseña la ciudadanía no es un acto que podría referirnos a indiferencia, ya que a través de ella se transmite una determinada lectura del mundo que permitirá a las futuras generaciones vincularse con los demás y proyectar de forma colectiva la construcción de una sociedad más justa y democrática. La escuela forma parte de este entramado ya que históricamente ha sido la encargada de dar vida a los lineamientos educativos para la formación de la ciudadanía. En una primera instancia, desde las concepciones cívicas que enfatizan la generación de valores y virtudes propias de una "buena ciudadanía", y luego, en una segunda instancia, en lo que se define como la formación ciudadana que promueve el respeto por la democracia y los derechos humanos.

En Chile la Formación Ciudadana forma parte del sistema educativo como un eje transversal, a partir de su integración como un proyecto educativo que debe ser abordado por las diferentes asignaturas que componen el currículo escolar. La participación en evaluaciones internacionales desarrolladas por la IEA en 2009 y 2016 evidenciaron que el estudiantado chileno obtiene resultados por debajo del promedio en conocimientos y actitudes hacia la ciudadanía (AGENCIA DE CALIDAD DE LA EDUCACIÓN, 2017, p. 33). Como una manera de hacer frente a esta problemática, y en medio de un álgido escenario político y social, en 2017 entró en vigencia la ley 20.911, que insta a los establecimientos educacionales reconocidos por el Estado a la elaboración de Plan de Formación ciudadana (PFC), acorde a sus respectivos contextos.

No obstante, los procedimientos que se han realizado en cada escuela para diseñar e implementar su PFC son poco conocidos. Si bien se han publicado informes acerca de su puesta en marcha (PNUD, 2018), son pocos los estudios que se han propuesto averiguar qué es lo que ocurre en la práctica con la educación ciudadana. Entre ellos es posible destacar la investiga ción desarrollada por Pinochet y Mercado (2019), en el marco de los procesos de acompañamiento realizados por la Universidad Católica del Norte a 25 escuelas y liceos de la Región de Antofagasta en el diseño del PFC. Como reflexiones finales, los autores apuntan a la necesidad de desarrollar un "perfil de docentes responsables del PFC". Señalan que deben ser personas empoderadas de su rol, con cierto manejo teórico y con experiencia en la promoción de la participación. Si bien estas condiciones parecen ser relevantes para una implementación exitosa del PFC, no hacen referencia a una cuestión fundamental: ¿para qué y cómo enseñar la ciudadanía?

Ante este contexto, la enseñanza de la educación ciudadana en Chile se estructura a partir de la integración de competencias y principios valóricos ligados a la formación democrática de los y las estudiantes. De acuerdo con los antecedentes aportados por informes y pruebas estandarizadas (IEA, 2009, PNUD, 2018), los procesos de aprendizaje de contenidos ligados a la educación ciudadana y el desarrollo de competencias que permitan al estudiantado asumir un rol activo en sociedad han tenido poco impacto en las instituciones escolares, evidenciando problemas educativos para la aplicación de los respectivos proyectos de Formación Ciudadana. 
En cuanto a los problemas específicos del desarrollo de la educación ciudadana, Cox y Castillo (2015) plantean que en Chile la investigación en torno a la enseñanza y práctica de la educación ciudadana es poca, estando la mayoría vinculada al análisis de las creencias y percepciones del profesorado. En definitiva, existe poca investigación relacionada a la implementación de los objetivos y programas curriculares elaborados para la formación ciudadana. Lo que se debe principalmente al hecho de que existe poca información sobre estrategias didácticas vinculadas a esta área de estudio.

Por esta razón, en el presente trabajo se analizan las finalidades y lineamientos metodológicos propuestos en el currículo de enseñanza obligatoria y la política educativa de educación para la ciudadanía en Chile. Es un análisis que pretende contribuir a esclarecer el perfil de ciudadanía que se quiere formar, los principios y valores que la inspiran, y el modo como se piensa su enseñanza. El propósito es aportar herramientas para la comprensión del estado actual de la Formación Ciudadana en Chile y favorecer el tránsito hacia una educación ciudadana en perspectiva crítica y democrática.

\title{
2 LA EDUCACIÓN PARA LA CIUDADANÍA EN EL CONTEXTO EDUCATIVO CHILENO
}

En las dos últimas décadas la formación ciudadana ha irrumpido en los sistemas educativos a nivel nacional e internacional. Su propósito ha sido vinculado con la superación de problemáticas sociales y políticas que tocan elementos esenciales del sistema democrático que se observa con fragilidad (BOLÍVAR, 2007, p. 43). La educación para la ciudadanía se introduce en los sistemas educativos en conjunto con los derechos humanos, como un binomio inseparable dirigido al fortalecimiento de la convivencia democrática.

La Formación Ciudadana en Chile mantiene una carga histórica importante en relación a su integración en los proyectos educativos del país y ha servido como estandarte de lucha frente a los constantes procesos de crisis política y social suscitados durante el siglo XX. De esta forma, el primer paso para la inserción de este ámbito educativo se dio a través de lo que se conoce como Educación Cívica, concepto ampliamente estudiado desde las disciplinas pedagógicas e historiográficas dedicadas al estudio del civismo y su utilización como herramienta eficaz para la construcción del discurso de identidad nacional, que da vida en sus inicios a la enseñanza de la historia como disciplina escolar.

En la reforma educacional de la década de 1990, se estableció que la Formación Ciudadana debía estar integrada de manera transversal a lo largo de toda la secuencia escolar y distribuida en distintas áreas de aprendizaje. Se incorporaron también los Objetivos Fundamentales Transversales que apuntaban al desarrollo de habilidades, valores y actitudes fundamentales para la vida democrática (BASCOPÉ; COX; LIRA, 2015, p. 247). Esta reforma postulaba una visión más amplia de la ciudadanía que la ofrecida por la tradicional Educación Cívica. Cox y García (2015) afirman que este cambio significó una expansión en tres dimensiones:

\begin{abstract}
Expansión temática porque el foco de los contenidos de conocimiento se amplía desde la institucionalidad política (nación, Estado, gobierno, ley) a problemáticas sociales, morales y medioambientales; 2) expansión cuantitativa, porque la presencia de la formación ciudadana es redefinida en forma sustancial: de estar ubicada al final de la secuencia escolar (últimos grados de la educación media) pasa a estar presente a lo largo de la misma; y 3) expansión formativa, al plantearse objetivos de aprendizaje que junto con el conocimiento se refieren a habilidades y actitudes(COX;GARCÍA, 2015, p 289).
\end{abstract}

En lo que va corrido del siglo XXI, esta tendencia se ha mantenido. El año 2009 se realizó un ajuste al currículum de educación ciudadana, en atención a las orientaciones contempladas en el Informe de la Comisión de Formación Ciudadana del año 2004. Entre otros lineamientos, destacan los asociados a fortalecer los aprendizajes sobre institucionalidad política democrática, la promoción de la participación civil, el desarrollo de una conciencia de derechos y deberes ciudadanos, el fomento a la cohesión social y el desarrollo de un sentido de pertenencia a la comunidad nacional (MARDONES, 2015). Como resultado de este ajuste, la presencia de la Formación Ciudadana en el currículum se densificó y fortaleció (COX ; GARCÍA, 2015, p.285).

Las Bases Curriculares de 2013 propendieron a la redefinición de la Formación Ciudadana mediante Rev. Espaço do Currículo (online), João Pessoa, v.13, n.2, p. 191-202, maio/agos. 2020. 
la inclusión de un eje específico en la asignatura de Historia, Geografía y Ciencias Sociales, desde Primer Año de Educación Básica hasta Segundo Año de Enseñanza Media. . Esto permitió definir con mayor precisión los Objetivos de Aprendizaje en los diferentes niveles de enseñanza. En abril de 2016 se promulgó la Ley $\mathrm{N}^{\circ} 20.911$, cuyo artículo primero obliga a que los establecimientos educacionales reconocidos por el Estado desarrollen un Plan de Formación Ciudadana (PFC) que integre y complemente las definiciones curriculares en esta materia (LEY $\left.N^{\circ} 20.911,2016\right)$. La legislación indica los propósitos que deben perseguir estos planes y entrega lineamientos generales respecto de su implementación. Estos objetivos se pueden organizar en base a tres criterios: conocimientos; valores y actitudes; y vida democrática (Tabla 1).

Tabla 1 - Organización de los objetivos contemplados en la Ley № 20.911

\begin{tabular}{lll}
\hline Conocimientos & Valores y actitudes & Vida democrática \\
\hline Concepto de ciudadanía. & Ciudadanía activa, & Garantizar el desarrollo de \\
Estado de Derecho. & Cumplimiento de derechos y & una cultura democrática y \\
Institucionalidad local, & deberes, valoración de la & ética en la escuela. \\
regional y nacional. & diversidad social y cultural & Participación de los \\
Derechos reconocidos en la & del país, & estudiantes en temas de \\
Constitución y en los & Transparencia y probidad. & interés público. \\
tratados internacionales & Tolerancia y pluralismo & Ejercicio de una ciudadanía \\
suscritos y ratificados por & & crítica, responsable, \\
Chile & & respetuosa, abierta y \\
& & creativa. \\
\hline
\end{tabular}

Fonte: Elaboración propri a partr de la Ley $\mathrm{N}^{\circ} 20.911$

En 2016 el Ministerio de Educación publicó un material denominado Orientaciones para la elaboración del Plan de Formación Ciudadana donde se sugieren herramientas de trabajo para que cada establecimiento educacional diseñe y ejecute su propio PFC, en vínculo con el currículum nacional, los proyectos educativos institucionales y los planes de mejoramiento educativo (MINEDUC, 2016a).

Durante el año 2017 en cumplimiento con las “disposiciones transitorias" de la Ley, el Ministerio de Educación diseñó una propuesta de bases curriculares donde se incorpora la asignatura de Educación Ciudadana en Tercer y Cuarto Año Medio. Su finalidad sería profundizar y enriquecer lo visto a lo largo de la trayectoria escolar, dando énfasis a "conocimientos, habilidades y actitudes que fortalezcan la participación ciudadana y la ética-política, la valoración y exigibilidad de los derechos humanos, y la capacidad de comprender y analizar desde los principios democráticos, la institucionalidad, el territorio y los modelos de desarrollo económico" (MINEDUC, 2017).

\section{LA EDUCACIÓN CIUDADANA DESDE UN POSICIONAMIENTO CRÍTICO: finalidades y metodologías de enseñanza}

Enseñar educación ciudadana y democracia exige una toma de posición respecto a las finalidades que se persiguen. Ubicarse en un enfoque crítico implica distanciamiento respecto otras racionalidades o tradiciones epistemológicas, como por ejemplo, la positivista-técnica y la humanista (BENEJAM, 1997). La primera prioriza finalidades educativas aparentemente objetivas y neutras, comúnmente al servicio del nacionalismo. La segunda se caracteriza por un enfoque paidocéntrico, donde el conocimiento emerge como una construcción mediada por las interpretaciones de las personas bajo la lógica del aprendizaje por descubrimiento (PAGÈS; SANTISTEBAN, 2014).

Como contrapartida, desde una racionalidad crítica toma relevancia la idea de formar ciudadanía para transformar la escuela en función del cambio social (CERDA; EGAÑA et al, 2004). Este supuesto concuerda con la idea de "educación problematizadora" propuesta por Freire (2012), que incentiva la reflexión sobre la realidad, para actuar sobre ella y transformarla. En torno a esta misma pretensión, Giroux (2015) sostiene que el profesorado en vez de moldear a sus estudiantes para que se vuelvan espectadores de la realidad debe contribuir a que se conviertan en agentes de cambio.

De esta manera, apoyados en los planteamientos teóricos de la pedagogía crítica, se asume la importancia de comprender la teoría como fundamento para intervenir en los contextos y el poder, 
capacitando a las personas para que participen en la búsqueda del bien colectivo (GIROUX, 2003). Por consiguiente, la educación ciudadana debe incluir la problematización de las relaciones sociales y el ejercicio del poder, buscando promover la transformación de los elementos que subyacen al modelo tradicional de educación y pedagogía.

Entendido así, un currículum crítico de educación ciudadana debe asumir una finalidad emancipadora, donde se priorice la formación de un pensamiento social dirigido a la acción y a la transformación de la realidad (PAGÈS, 1994). En esta misma línea, ROSS (2001) argumenta que la enseñanza crítica de las ciencias sociales reclama el planteamiento y el análisis de problemas con miras a la comprensión y a la transformación del mundo. Agrega que enseñar Ciencias Sociales no puede reducirse a la implementación de un currículum diseñado desde las élites, sino que comporta el desafío de formar para comprender cómo es el mundo y cómo se podría actuar para cambiarlo.

Que el aprendizaje forme parte del proceso de cambio social es uno de los más importantes proyectos de la pedagogía crítica (CASTILLO, 2003). Lo anterior invita a las y los docentes a la toma de posición frente a lo político, empujándoles a posicionarse y ser agentes de cambios estructurales. En su dimensión transformadora, la pedagogía crítica presenta la formación ciudadana como un camino que permite estimular las pasiones, imaginaciones e intelectos, para que las y los estudiantes sean movidos a desafiar a las fuerzas sociales, políticas y económicas existente (GIROUX 1998, apud CERDA; EGAÑA et al, 2004). Lo anterior es definido por estos autores como coraje cívico, un modo emancipador de la educación ciudadana.

Siguiendo a CERDA, EGAÑA et al (2004), la propuesta de una educación para la ciudadanía desde la pedagogía crítica, enfatiza en la participación de las y los estudiantes durante el proceso educativo, para lo cual es vital el desarrollo de habilidades de pensamiento crítico, reconociendo como parte de sus biografías, las fuerzas estructurales e ideológicas que influyen en sus vidas.

En síntesis, puede señalarse que la educación ciudadana desde una concepción crítica asume las siguientes finalidades: comprender los problemas sociales y el funcionamiento del poder, desarrollar el compromiso social y político de la juventud, desafiar las injusticias, imaginar futuros alternativos y actuar para transformar la realidad.

Desde la Didáctica de las Ciencias Sociales se han analizado las ventajas de adoptar un enfoque centrado en el estudio de problemas sociales (EVANS, 2001; PAGÈS, 2007; PAGÈS; SANTISTEBAN, 2014; CANALS, 2018). Esta alternativa se vincula a la idea de "educación problematizadora" acuñada por FREIRE (2012) y al ejercicio crítico de la pedagogía que defiende Giroux (2019), a partir del tratamiento de preguntas y problemas sociales cruciales.

Pagès (2007) concibe este enfoque como una gran oportunidad para la educación ciudadana, porque acerca el mundo a las y los estudiantes y les prepara para la vida. Añade que constituye una apuesta por una enseñanza más crítica de las Ciencias Sociales que incentiva a la juventud a asumir protagonismo en la construcción de su realidad presente, y decidir con libertad su propio futuro y el del mundo.

Es un enfoque que prioriza entre sus finalidades la formación para una ciudadanía democrática. Asimismo, contribuye al desarrollo de un pensamiento social y crítico, para promover la participación de las y los jóvenes en la construcción del futuro (PAGÈS; SANTISTEBAN, 2011). González-Monfort (2011), agrega que esta perspectiva se basa en la intención de trabajar situaciones sociales conflictivas, candentes o controvertidas, generando entre el estudiantado reflexión, preguntas y debates abiertos sobre la actualidad.

Apuntando al mismo desafío, en la tradición crítica de los Estudios Sociales se ha hecho hincapié en la relevancia de seleccionar temas controvertidos -controversial issues- para favorecer la formación de competencias ciudadanas (Levstik; Tyson, 2008). Hess (2008) concibe esta estrategia como una herramienta clave para preparar a la juventud en la participación plena, competente y auténtica en el mundo. Añade que la discusión sobre asuntos públicos en el aula es fundamental para fortalecer el 
compromiso democrático. No obstante, indica que no se trata de la discusión per se, sino entendida como un dispositivo didáctico para lograr objetivos mayores, como la comprensión y compromiso con los valores democráticos, el incremento del pensamiento crítico y el desarrollo de habilidades interpersonales.

Otro camino posible es el trabajo con conflictos sociales. Bickmore (2008) argumenta que una educación que tenga como finalidad la democratización y el cambio social, debe sacar a la luz los conflictos existentes en la sociedad. En su perspectiva, el conflicto no se reduce al ejercicio de la violencia o la represión. Se trata más bien de situaciones donde está en juego la lucha contra las injusticias sociales. Desde una postura similar, López (2011) defiende la idea de trabajar "conflictos sociales candentes". Este concepto hace alusión a asuntos que dividen a la sociedad y generan confrontación. Al respecto, apunta que no es suficiente con que las y los estudiantes aprendan a resolver conflictos, sino que es necesario que comprendan por qué se originan y cómo gestionarlos democráticamente.

En el ámbito de las estrategias educativas, East y Evans (2010) concluyen que las metodologías didácticas participativas, democráticas e inclusivas, se han transformado en una necesidad profunda e inminente para todo sistema educativo que tenga por objetivo la promoción del conocimiento y el compromiso ciudadano.

Frente a este desafío es relevante puntualizar en la existencia de diversas formas de comprender y enseñar la formación ciudadana, las cuales, podrían remitir a diferentes concepciones, inflexiones o énfasis en torno a lo que debe ser el rol de la institución escolar: las concepciones de sujeto, democracia y ciudadanía. Cerda, Egaña, Magendzo, Santa Gruz y Varas (2004) identificaron cuatro modos o maneras sobre las que se concibe y practica la formación ciudadana desde lo pedagógico:

Tabla 2 - Tipos de práctica en Formación Ciudadana

\begin{tabular}{|c|c|}
\hline $\begin{array}{l}\text { a) Formación para la ciudadanía } \\
\text { politica }\end{array}$ & $\begin{array}{l}\text { - Concepciones liberales y constitucionalistas. } \\
\text { - Vinculada a la tradición de la educación civica. }\end{array}$ \\
\hline $\begin{array}{l}\text { b) Formación para la ciudadanía } \\
\text { social }\end{array}$ & $\begin{array}{l}\text { - Derechos civiles y políticos. } \\
\text { - Derechos económicos, culturales y medioambientales. } \\
\text { - Noción omnicomprensiva del ser humano y la ciudadanía. }\end{array}$ \\
\hline $\begin{array}{l}\text { c) Forma para una ciudadania } \\
\text { activa }\end{array}$ & $\begin{array}{l}\text { - Énfasis en la participación de los ciudadanos. } \\
\text { - Auto construcción de la sociedad. } \\
\text { - Autonomía/ autogobierno comunitarista } \\
\text { - Desarrollo del capital social. }\end{array}$ \\
\hline d) Formación ciudadana crítica & $\begin{array}{l}\text { - Problematización de las relaciones sociales. } \\
\text { - Discusión de las relaciones de poder. } \\
\text { - La escuela en función del cambio social. } \\
\text { - Desafiar las fuerzas sociales y politicas "Coraje cívico". }\end{array}$ \\
\hline
\end{tabular}

Fonte: Adaptado, Cerda, Egañs, Magendzo e otros (2004)

Las cuatro formas de comprender y enseñar la ciudadanía antes mencionadas, transitan desde principios políticos y filosóficos en las visiones liberales y las diferentes perspectivas sociales desarrolladas por corrientes críticas de la pedagogía y la filosofía política. Estas perspectivas desarrollan un modelo de ciudadanía a través de la incorporación en su estudio de elementos problematizadores de los procesos sociales, posibilitando una lectura más comprensible de lo que es la relación existente entre los derechos humanos y la ciudadanía, argumentando que no es posible pensar en los derechos de las personas basadas exclusivamente en lo constitucional y lo político.

Coz y Castillo (2015) analizan las prácticas pedagógicas desde una doble matriz: primero lo expuesto por John Dewey en sus estudios clásicos a favor de la inclusión de prácticas pedagógicas que desarrollen habilidades de investigación, como el trabajo grupal y el estudio de problemas políticos y sociales reales, 
que permitan a los estudiantes desarrollar aspiraciones democráticas en la educación (DEWEY, 1916 apud COX, CASTILLO, 2015, p.43). En segundo lugar, las afirmaciones de Gainous y Martens (2012), quienes profundizan en la importancia de las variaciones en los métodos de instrucción cívica para desarrollar capacidades democráticas en los estudiantes.

Como una manera de definir un tipo de ciudadanía y de Educación Ciudadana que se aleja de las finalidades y estrategias de enseñanza más próximas a una perspectiva crítica, en este artículo utilizamos el concepto ciudadanía de puertas adentro. Mediante este término buscamos referir a un tipo de ciudadanía que permanece encerrada en los establecimientos educativos con baja predisposición a la intervención social. Se trata de un tipo de ciudadanía que no asume como horizonte político la transformación de la sociedad. Por consiguiente, educar para una ciudadanía de puertas adentro implica priorizar el desarrollo de habilidades de discusión, debate y deliberación al interior de una sala de clases, en lugar de aprendizajes dirigidos a formar un estudiantado crítico, comprometido socialmente y con capacidad para actuar en favor del cambio social.

\section{SOBRE LA METODOLOGÍA}

Los resultados aquí expuestos forman parte de un corpus de estudio cualitativo, realizado a partir del análisis de las finalidades y las metodologías de enseñanza descritas en el currículo oficial de Formación Ciudadana en Chile. Como técnica de recogida de información se utilizó el análisis documental con la intención de interpretar, analizar y sintetizar el texto de tal manera que posibilite el levantamiento de categorías analíticas que permitan transitar de forma constante entre la teoría y la visión de quienes investigan (EIROA, 2009, p.8).

En este caso, interesa particularmente analizar desde el texto íntegro las perspectivas y corrientes pedagógicas e ideológicas que subyacen a la idea de Formación Ciudadana planteada desde la política educativa chilena y los planteamientos metodológicos y didácticos propuestos para trabajar en las aulas.

\section{DISCUSIÓN Y RESULTADOS}

En este apartado se presentan los resultados de un estudio como análisis de la documentación oficial de Formación Ciudadana en Chile. En primer lugar, se dan cuenta de las finalidades atribuidas a la Formación Ciudadana en el currículum oficial. En segundo lugar se expone el análisis referido a las estrategias de enseñanza.

\subsection{Las finalidades atribuidas a la Formación Ciudadana}

La revisión y análisis de documentación oficial da cuenta de la existencia de tres ejes que permiten comprender las finalidades atribuidas a la Formación Ciudadana: los valores o principios que inspiran la ciudadanía; las competencias que se consideran pertinentes para el desarrollo de la ciudadanía; el perfil de estudiantes que se aspira formar.

En torno a los valores y principios que inspiran la ciudadanía, se mencionan específicamente el respeto por los Derechos Humanos y la valoración de la democracia como sistema político y social. Se señala que ambos son tópicos claves que "emergen como organizadores de todo trabajo vinculado a la Formación Ciudadana” (MINEDUC, 2016b, p.33). También se ofrece una definición que se repite en los distintos documentos:

La formación ciudadana corresponde a un proceso formativo continuo que permite que los niños, niñas, jóvenes y adultos desarrollen un conjunto de conocimientos, habilidades y actitudes que resultan fundamentales para la vida en una sociedad democrática. Busca promover en distintos espacios, entre ellos las comunidades educativas, oportunidades de aprendizaje que permitan que niños, niñas, jóvenes y adultos se formen como personas integrales, con autonomía y pensamiento crítico, principios éticos, interesadas en lo público, capaces de construir una sociedad basada en el respeto, la transparencia, la cooperación y la libertad (MINEDUC 2017, p.45)

A partir del fragmento anterior es posible advertir que el propósito final que se atribuye a la Formación Ciudadana es la construcción de una sociedad democrática basada en principios de 
convivencia. Por tanto, es válido preguntarse ¿cuál es la concepción de democracia que se propone y cuáles son los aprendizajes que se espera aprendan los niños, niñas y jóvenes del país? En cuanto a la concepción de democracia, se identifica un marcado énfasis en el reconocimiento de los procesos sociales e históricos que dan vida y sustento al sistema democrático representativo. Al mismo tiempo, se exacerba la democracia como una forma de organización política que permite la convivencia social pacífica y el resguardo de los derechos fundamentales de los seres humanos.

En relación al objetivo de reconocer el sistema democrático y los Derechos Humanos en el marco de un correlato histórico, se posicionan discursos a partir de un devenir social fluctuante, en constante transformación, haciendo especial énfasis en que la democracia es un modelo político que necesita ser revisado, reflexionado y construido de manera constante, tal como lo menciona el propio programa de Formación Ciudadana al decir que "interesa que comprendan que la democracia se construye a diario y que, así como es perfectible, está expuesta a diversas situaciones que la ponen en riesgo" (MINEDUC, 2016b, p.34).

Es así como se postula la democracia en el horizonte formativo de la educación ciudadana. Se hace hincapié en que constituye tanto un sistema de organización política, como un sistema social. En efecto, en los objetivos de la Ley 20.911 estas dimensiones de la democracia también son explícitas. Esto es clave para comprender cómo se definen las competencias ciudadanas y el perfil del estudiantado.

De manera frecuente se señala que el propósito es contribuir a la formación de una ciudadanía activa, participativa, comprometida con la sociedad y la democracia. En las "Orientaciones Curriculares para el desarrollo del Plan de Formación Ciudadana" (MINEDUC, 2016, p. 24) se define un perfil de estudiante "informado e interesado en los asuntos comunes, cuyas prácticas participativas se expresan en distintos espacios" (p.24). Las competencias que se sugieren son tres: "la participación activa, el análisis crítico, y el respeto y la consideración de las opiniones distintas" (p.105). Se hace énfasis en la importancia de la participación, considerada clave para el logro de una convivencia democrática. Se argumenta que el aprendizaje de la ciudadanía requiere el desarrollo de habilidades que permitan el involucramiento activo de los estudiantes, tales como la argumentación y la deliberación.

\subsection{La enseñanza de la ciudadanía}

En todos los documentos donde el MINEDUC (2016b) formula propuestas prácticas es posible hallar afirmaciones alusivas al tipo de didáctica que debiese emplearse al enseñar ciudadanía. El documento donde se describe con mayor detenimiento esta perspectiva es el denominado "Orientaciones Curriculares para el desarrollo del Plan de Formación Ciudadana" (MINEDUC, 2016b). Allí se sugiere realizar "un trabajo didáctico centrado en las y los estudiantes" (p. 38) por lo que propone emplear metodologías de enseñanza "interactivas". La justificación que brinda para la elección de esta perspectiva se resume en el siguiente fragmento:

"Estrategias de este tipo son útiles también porque, al exigir a las y los estudiantes que
tomen parte en las actividades educativas, les entregan una base formativa para la par-
ticipación social argumentando, expresando opiniones, buscando y discriminando infor-
mación, dialogando con otras y otros respecto a puntos de vista distintos al propio,
dando a conocer sus ideas y propuestas sobre un tema o problema y, sobre todo, acor-
dando con otros las formas de trabajo" (MINEDUC, 2016b, p. 39).

También hace referencia al tipo de actividades que permitirían concretar este enfoque:

Algunas de las actividades más comunes que pueden realizarse para trabajar estrategias de enseñanza interactivas son dramatizaciones, teatro foro, trabajo con proyectos, estudios de casos, juegos de roles, debates, dilemas morales, simulaciones, aprendizaje basado en problemas y aprendizaje basado en servicio. (MINEDUC, 2016b, p. 40).

En relación a las temáticas que debiesen ser abordadas, el MINEDUC (2016b) indica lo siguiente: "Se propone focalizar el trabajo en temas controversiales o de interés público, de manera de enfrentar y abordar conflictos y abrir espacios diversos de participación". (MINEDUC, 2016b, p. 40). En síntesis, podría señalarse que la perspectiva didáctica declarada por el MINEDUC (2016b) reúne dos condiciones: 1) emplear "metodologías interactivas" que favorezcan la participación del estudiantado; y, 2) el trabajo 
ISSN $1983-1579$

Doi: 10.22478/ufpb.1983-1579.2020v13n1.51539

http://periodicos.ufpb.br/ojs2/index.php

con temáticas controvertidas o de interés público.

\section{CONCLUSIONES}

Esta investigación surgió con el propósito de analizar las finalidades y metodologías de enseñanza de la Formación Ciudadana en el contexto educativo chileno, a partir de una mirada al currículum oficial. En términos generales es posible señalar que la educación ciudadana es entendida en vínculo con la democracia y la participación. Sin embargo, los supuestos que subyacen a dichas ideas no son del todo evidentes.

En primer lugar, el MINEDUC declara que los principios centrales de la ciudadanía son el compromiso con la democracia y el respeto por los Derechos Humanos. Este es un posicionamiento que se ajusta a las finalidades que desde la teoría se asignan a la educación para la ciudadanía, entendida como una formación para la democracia. Sin embargo, no se alude a propósitos que asocien la Formación Ciudadana con un horizonte político de transformación de la realidad.

En relación a las competencias, la participación activa y el desarrollo del pensamiento crítico son las que se aluden con mayor frecuencia. Habilidades relacionadas con la reflexión, el diálogo, la argumentación y la deliberación parecen ser las predilectas. Estos planteamientos se aproximan a las competencias definidas desde la teoría para la formación de una ciudadanía crítica y democrática. Sin embargo, no hacen referencia a la idea de intervenir en la realidad, elemento considerado fundamental desde la teoría crítica.

Algo similar ocurre con el perfil de estudiantado. EI MINEDUC declara que su intención es contribuir a la formación de un estudiantado participativo y comprometido con la sociedad. Sin embargo, esa participación y compromiso no puede ser condescendiente con el status quo y debe orientarse a exponer, desafiar y combatir las injusticias sociales, proponiendo futuros alternativos. Sin sorpresas se evidencia que esta es una idea que no se menciona en las propuestas oficiales.

En relación a las estrategias de enseñanza, el posicionamiento didáctico del MINEDUC plantea la idea de utilizar "metodologías interactivas" y tratar asuntos de interés público, temas controvertidos y conflictos. A simple vista este posicionamiento parece estar próximo al estudio de problemas sociales. Sin embargo, cuando se revisan las propuestas prácticas para su materialización se constata que prevalece la idea de discutir y debatir estos temas al interior de la sala de clases, eludiendo el espacio público y la acción social.

Frente a esta información es preciso preguntarse: ¿es suficiente discutir y debatir los problemas sociales, si el propósito es transformar la realidad y contribuir al fortalecimiento de la democracia y la justicia social?

Esta información permite postular que desde el MINEDUC se promueve un tipo de ciudadanía que podríamos definir como ciudadanía de puertas adentro. Una ciudadanía que se caracteriza por discutir y debatir temas de interés público, dentro de los límites que se le imponen; ciudadanos y ciudadanas respetuosas de la diversidad de opiniones, con capacidad para llegar acuerdos, pero incapaces de romper con lo establecido. Es una ciudadanía que reflexiona, es consciente de que en el mundo existen problemas y conflictos, pero no interviene para solucionarlos o avanzar hacia un cambio social. Es, en definitiva, una ciudadanía respetuosa con el orden establecido, inofensiva, si se le compara con la idea de ciudadanía peligrosa propuesta por Ross y Vinson (2012).

Esta constatación coincide con la señalada en la investigación de Gonzá-lez-Valencia y Santisteban (2016) sobre el estado actual de la Formación Ciudadana en Colombia. Según plantean los autores, en dicho sistema educativo la educación ciudadana ha sido vaciada de contenido político y se ha privilegiado una racionalidad instrumental que reduce el comportamiento ciudadano a procedimientos de resolución de conflictos entre personas, sin ahondar en temas de mayor alcance político como las desigualdades sociales y la exclusión. Los autores -citando a Restrepo (2009)- apuntan el concepto de Formación Ciudadana "agorafóbica", que reduce todo a conductas individuales y evade la discusión pública. 
Lo anterior nos permite sugerir como camino para la mejora de los procesos de enseñanza y aprendizaje de la ciudadanía, la utilización de estrategias didácticas que promuevan la intervención. Estas últimas emergen como alternativa válida para potenciar la participación y el compromiso ciudadano de las y los estudiantes, ajustando la enseñanza de la ciudadanía con propósitos dirigidos a la participación activa, la búsqueda del cambio social y en definitiva, el fortalecimiento de la democracia.

\section{Referencias}

AGENCIA DE CALIDAD DE LA EDUCACIÓN. Estudio Internacional de Educación Cívica y Formación Ciudadana. Presentación nacional de resultados. Santiago: Agencia de Calidad de la Educación, 2017.

BASCOPÉ, Martín; COX, Cristian; LIRA, Robinson. Tipos de ciudadanos en los currículos del autoritarismo y la democracia. En COX, Cristian y CASTILLO, Juan Carlos (Edit). Aprendizaje de la ciudadanía: contextos, experiencias y resultados. Santiago de Chile: Ediciones UC, 2015. p. 245-281.

BENEJAM, Pilar. Las finalidades de la educación social. En BENEJAM, Pilar; PAGÉS. Joan (Edits). Enseñar y aprender Ciencias Sociales, Geografía e Historia en la educación secundaria. Barcelona: ICE/ Horsori, 1997. p. $33-52$.

BICKMORE, Kathy. Social justice and the social studies. En LEVISTIK, Linda; TYSON, Cynthia. Handbook of research in social studies education. New York; London: Routledge, 2008. p. 155-171.

BOLÍVAR, Antonio. Educación para la ciudadanía: algo más que una asignatura. Barcelona: Ediciones Graó, 2007.

CANALS, Roser. L'ensenyament i l'aprenentatge de les ciències socials avui: un enfocament disciplinari o a partir de problemes? Revista Catalana de Pedagogia, 2018. p. 63-87.

CASTILLO, Juan Carlos. La formación de ciudadanos en la escuela: un escenario posible. Revista Latinoamericana de Ciencias Sociales, niñez y juventud, v.1, n 2, 2003, p. 1-19.

CERDA, Ana María; EGAÑA, Loreto; MAGENDZO, Abraham, SANTACRUZ, Eduardo; VARAS, Rene. El complejo camino de la formación ciudadana: una mirada a las prácticas docentes. Santiago de Chile: LOM ediciones, 2004.

COX, Cristián; CASTILLO, Juan Carlos. Aprendizaje de la ciudadanía: contextos, experiencias y resultados. Santiago de Chile; Ediciones UC, 2015.

EAST, Viv; EVANS, Linda. Guía práctica de necesidades educativas especiales. Madrid: Ediciones Morata, 2010.

EIROA, Matilde. Los métodos de las ciencias sociales y la investigación histórica Hispania Nova. Revista de Historia Contemporánea, n 9, 2009, p. 77-91.

EVANS, Ronald. Teaching social Issues; Implementing an Issues- Centered Curriculum. En ROSS, Wayne (Ed), The social Studies curriculum: purposes, problems and posibilities. New York: State University of New York, 2001. p. 291-309.

FREIRE, Paulo. Pedagogía de la autonomía. México: Siglo XXI. 2006.

. Pedagogía del oprimido. Madrid: Siglo XXI. 2012.

GAINOUS, Jason; MARTENS, Allison. The effectiveness of civic education: Are "good" teachers actually good for "all" students? American Politics Research, v. 40, n. 2, 2012 232-266.

GIROUX, Henry. Pedagogía y política de la esperanza. Teoría, cultura y esperanza. Buenos Aires: Amorrortu editores. 2003.

- Pedagogías Disruptivas y el Desafío de la Justicia Social bajo Regímenes Neoliberales. Revista Internacional de Educación para la Justicia Social (RIEJS), v. 4, n. 2, 2015. p. 13-27.

. Hacia una pedagogía de la esperanza educada bajo el capitalismo de casino. Pedagogía y Saberes, s/v, n. 50, 2019. p. 153-158. 
ISSN $1983-1579$

Doi: 10.22478/ufpb.1983-1579.2020v13n1.51539

http://periodicos.ufpb.br/ojs2/index.php

GONZÁLEZ-MONFORT, Neus. Reflexions al voltant de les qüestions socialment vives i l'ensenyament de les ciències socials. Conclusions de les VII Jornades Interinacionals de Recerca en Didàctica de les Ciències Socials. En PAGÉS, joan; SANTISTEBAN, Antoni (Edits), Les qüestions socialment vives i l'ensenyament de les ciències socials. Bellaterra: Universitat Autònoma de Barcelona, 2011. p. 153-161.

GONZÁLEZ-VALENCIA, Gustavo; SANTISTEBAN-FERNÁNDEZ, Antoni. La formación ciudadana en la educación obligatoria en Colombia: entre la tradición y la transformación. Educ.Educ. v.19, n. 1. 2016, p. 89-102

HERNANDO, Jairo Gómez. Aprendizaje ciudadano y formación ético-política. Editado por Universidad distrital Francisco José Caldas, 2005.

LEVSTIK, Linda; TYSON, Cynthia (Edits). Handbook of Research in Social Studies Education. New York; London: Routledge. 2008.

LÓPEZ, Ramón. Conflictos sociales candentes en el aula. En PAGÉS, Joan, SANTISTEBAN, Antoni (Edits) Les qüestions socialment vives i l'ensenyament de les ciències socials. Bellaterra: Universitat Autònoma de Barcelona, p. 65-76. 2011.

MINEDUC,. Orientaciones para la elaboración del Plan de Formación Ciudadana. Santiago: Ministerio de Educación, 2016a.

. Orientaciones curriculares para el desarollo de Plan de Formación Ciudadana. Santiago, Chile: Ministerio de Educación. Unidad de Currículum y Evaluación, 2016b

. Orientaciones para la participación de las comunidades educativas en el marco del Plan de Formación Ciudadana. Santiago: Ministerio de Educación de Chile, 2017.

MUÑOZ, Pablo, GAMBOA, Aloiso y URBINA, Ernesto. Formación ciudadana: discursos de educadores y estudiantes sobre las actitudes y el pensamiento crítico orientados al ejercicio de la ciudadanía. Revista interamericana de Investigación, Educación y Pedagogía, v. 7, n. 1, 2014.p. 125-147,

PAGÉS, Joan. La enseñanza de las Ciencias Sociales y la educación para la ciudadanía en España. Revista Didáctica geográfica, s/v, n. 9, 2007. p. 205-214.

- La Didáctica de las Ciencias Sociales, el currículum de historia y la formación del profesorado. Signos. Teoría y práctica de la educación, s/v, n. 131994, p. 38-51.

PAGÉS, Joan; SANTISTEBAN, Antoni. Les qüestions socialment rellevants a l'ensenyament de les ciències socials a Catalunya: passat, present i futur. En PAGÉS, Joan; SANTISTEBAN, Antoni (Edits) Les qüestions socialment vives i l'ensenyament de les ciències socials. Bellaterra: Universitat Autònoma de Barcelona, 2011, p. 77-91,

PAGÉS, Joan; SANTISTEBAN, Antoni. Una mirada desde el pasado al futuro en la Didáctica de las Ciencias Sociales. En PAGÉS, Joan; SANTISTEBAN, Antoni (Edits) Una mirada al pasado y un proyecto de futuro. 25 años de investigación e innovación en Didáctica de las Ciencias Sociales. Vol1, Barcelona, Universidad Autónoma de Barcelona. p. 17-39, 2014.

PINOCHET, Sixtina; MERCADO, Javier. Reflexiones en torno a la implementación del Plan de Formación Ciudadana en la región de Antofagasta: perspectivas de los equipos responsables. En ORELLANA, Cristian; SALAZAR, Rodrigo; HASSE, Viviane (Edits.). Formación ciudadana en el contexto escolar. Conceptualización, avances y experiencias. Santiago: RIL Editores, 2019. p. 221-240,

PNUD. Estudio sobre la puesta en marcha del Plan de Formación Ciudadana. Santiago: Programa de las Naciones Unidas para el Desarrollo, 2018.

REDÓN, Silvia. La escuela como espacio de ciudadanía. Estudios pedagógicos, v. XXXVI, n. 2, 2010. p. 233259.

ROSS, Wayne. Social Studies Teachers and curriculum. En ROSS, Wayne (Ed), The social Studies curriculum: purposes, problems and posibilities. New York: State University of New York, 2001. p. 3-15. 
OBJETIVOS E METODOLOGIAS DE ENSINO PARA A EDUCAÇÃO CIDADÃQUINTANA-SUSARTE, S.; HIDALGO, A. R. ROSS, Wayne; VINSON, Kevin. La educación para una ciudadanía peligrosa. Enseñanza de las Ciencias Sociales, s/v, n. 11, 2012. p. 73-86.

Recebido em: 30/03/2020

Alterações recebidas em: 22/04/2020

Aceito em: 22/04/2020

Publicado em: 22/04/2020 